\title{
The Processing and Mechanical Performance of Cellulose Nanofiber-based Composites ${ }^{\dagger}$
}

\author{
Antonio Norio Nakagaito ${ }^{1}{ }^{*}$, Hitoshi Takagi ${ }^{1}$ and Jitendra Kumar Pandey ${ }^{1}$ \\ ${ }^{1}$ Advanced Materials Division, Institute of Technology and Science, The University of Tokushima, Tokushima 770-8506, Japan
}

(Manuscript Received September 27, 2011; Revised October 22, 2011; Accepted December 3, 2011)

\begin{abstract}
Nanocomposites based on cellulose nanofibers have been studied for a considerable time since its first introduction, however real applications seem to have hardly developed to these days. The high-strength of cellulose nanofibers suggests the potential to reinforce plastics to produce composites for semi-structural or even structural applications. This paper discusses some of the attempts to produce such high-strength nanocomposites and the main challenges that have to be overcome to bring them into commercial products.
\end{abstract}

Keywords: Cellulose nanocomposites, Cellulose nanofibers, Green nanocomposites

\section{Introduction}

Cellulose nanofibers are mostly found in nature in the cell wall of plant fibers, acting as the framework of a biocomposite made of matrix substances lignin and hemicelluloses. These nanofibers have lateral dimensions of a few nanometers and are comprised of a bundle of cellulose molecular chains arranged parallel to the longitudinal direction of the nanofibers. The crystalline portions have a Young's modulus of $138 \mathrm{MPa}$ [1], with the theoretical tensile strength predicted to be about $10 \mathrm{GPa}$ [2], but as the molecules are aligned along the axial direction of the nanofibers even in the amorphous regions, the real strength is estimated to be over 2 GPa. That is to say that the mechanical properties of cellulose nanofibers approach those of aramid fibers, making these natural nanofibers a sustainable alternative to high-strength synthetic fibers.

The first attempt to exploit cellulose nanofibers in composites was probably made by Boldizar et al. in 1987 [3], as stated by Berglund [4]. In this pioneering study, prehydrolyzed pulp fibers were sub-

\footnotetext{
"Corresponding author. Tel.: +81-88-656-7364, Fax.: +81-88-656-7093.

E-mail address: norio@me.tokushima-u.ac.jp

Copyright (c) KSOE 2011.
}

jected to mechanical treatments by a beater or a high-pressure homogenizer to extract the nanofibers, and subsequently compounded with thermoplastic polymers and injection molded. Even though the modulus of the composites increased significantly in relation to the neat matrixes, the tensile strength did not, or just decreased. This was probably due to agglomerations of the hydrophilic cellulose nanofibers, which are extremely difficult to disperse in the hydrophobic melted polymer. Years later, in 1995, cellulose whiskers extracted by acid hydrolysis from natural cellulosic sources, were composed with elastomers and the reinforcement mechanism was studied by researchers in France. Favier et al. $[5,6]$ mixed copolymerized styrene and butyl acrylate latex with an aqueous suspension of cellulose whiskers, and casted films by water evaporation. As the whiskers were kept in a water medium during processing, they were homogeneously dispersed in the composite film, and a $6 \mathrm{wt} \%$ whisker load resulted in a shear modulus in the rubbery state of over two orders of magnitude increase. And differently from the modulus of the matrix, which decreases with temperature, the modulus of the composite stayed constant up to the temperature of cellulose decomposition. The remarkable reinforcing 
effect and the unchanged modulus regardless of temperature increase, led the authors to assume that the whiskers formed a percolated network by bridging the hydroxyl groups of cellulose through hydrogen bonds. Percolated systems have all the reinforcing elements interconnected, forming an unbroken cluster spanning the whole material. It is like a stiff framework supporting the matrix rather than a myriad of individual reinforcing elements. In later studies, it was found that the bonding in percolated systems of cellulose nanofibers delivered better reinforcement than whiskers, when compared with the same matrix material [7]. This early finding, and a posterior systematic study [8] led to the conclusion that besides hydrogen bonds, cellulose nanofibers were interconnected by physical entanglements, due to the enhanced flexibility compared to whiskers. It was an indication that cellulose nanofibers, although not entirely crystalline as whiskers are, were capable of producing better reinforcing systems through percolation.

\section{High-strength cellulose nanocomposites}

\subsection{Rotor specification}

The phenomenon of percolation was later exploited by Yano and Nakahara [9], by producing molded materials using exclusively cellulose nanofibers and water. The nanofiber slurry was slowly compressed inside a mold while the excess of water was carefully removed by a suction pump. During the removal of water, capillary forces drew the nanofibers close enough to form hydrogen bonds, so that when completely dried the material became extremely compact and rigid. It could be regarded as a thick version of paper, but made of nano-sized fibers instead of micro-sized ones. The huge surface area difference between the two morphologies creates a proportionately higher number of hydrogen bonds, which makes a very strong material. The bending strength achieved a remarkable value of $250 \mathrm{MPa}$ and increased to $310 \mathrm{MPa}$ when $2 \mathrm{wt} \%$ oxidized starch was added to the initial slurry, as it acted as a plasticizer and produced a tougher material.

Based on the concept of preserving the percolated framework of cellulose nanofibers, paper-like sheets were fabricated by filtration of nanofiber aqueous suspensions and impregnated with phenolc resin and compression molded [10]. As shown in Fig. 1, if compared to composites made with the original kraft pulp fibers before converting into nanofibers, the bending modulus was slightly improved from around $18 \mathrm{GPa}$ to $19 \mathrm{GPa}$, however due to a higher strain at fracture, the nanocomposites had the bending strength of about $370 \mathrm{MPa}$ against $250 \mathrm{MPa}$ of the microcomposites. The highly extended surface area of the nanofibers creates an increased bond density due to the nano-scalar dimensions, so that fracture sites are smaller and widely distributed in the material volume, delaying failure and consequently increasing strength.

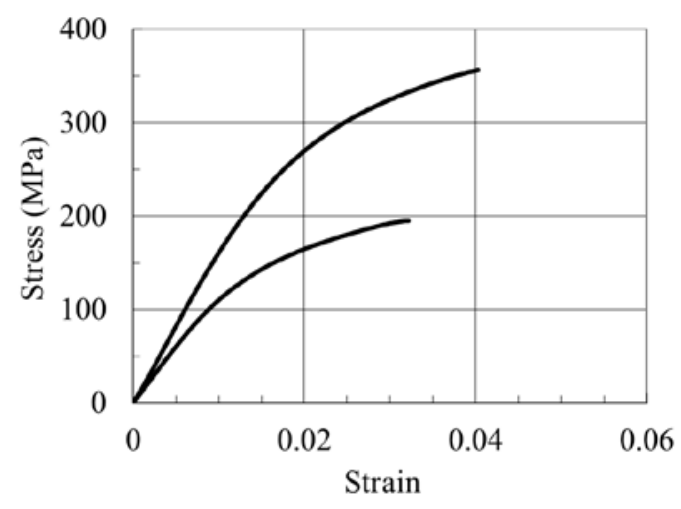

Fig. 1. Typical flexural stress-strain curves of kraft pulp fiber/phenolic resin microcomposite (a) (resin content: 9.7wt\%) vs. cellulose nanofiber/phenolic resin nanocomposite (b) (resin content: 9.8wt\%).

If the change in mechanical properties is analyzed as a function of the degree of fibrillation, i.e., differing morphologies in between the microfibers and nanofibers, the transition is not gradual. It actually occurs in a stepwise fashion instead. The bending strength of composites made with varying cellulose morphologies, from the original kraft pulp all the way through the intermediate degrees of fibrillation, up to the nanofibers was analyzed [11]. Up to a certain point of fibrillation, the strength of the composites is unchanged. The observation of the morphology by scanning electron microscopy revealed that the fibrillation is restricted to the surface of the fibers, which still keeps the micro-scale structure intact. When the cell wall is broken and 
converted into thin fibril bundles, the strength increases abruptly, increasing linearly from that point as the fibrillation progresses. This result showed the necessity to completely convert the fibers into nanofibers in order to obtain any gains in strength. Even when considering cellulose nanofibers, slight differences in morphology can affect the strength of the final composites. This was demonstrated by Takagi [12] by using two different grades of commercially available cellulose nanofibers, a coarser type and a finer one. Completely green composites were fabricated with polylactic acid resin, and the results of tensile test were as shown in Fig. 2. The presence of thick nanofiber bundles in the coarser cellulose morphology acted as defects that reduced the ultimate strength of composites, implying that the uniformity in width of the nanofibers is critical to produce high-strength composites.

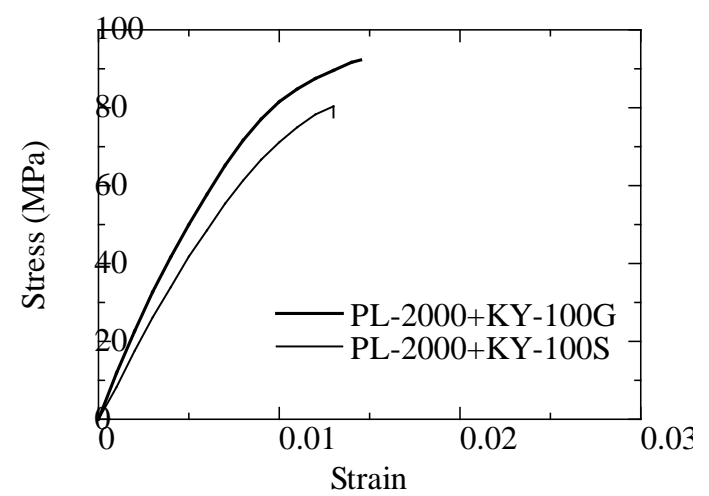

Fig. 2. Typical tensile stress-strain curves of polylactic acid reinforced with cellulose nanofibers with different degrees of fibrillation: coarse (a) and fine (b) morphologies (fiber content of both materials: $50 \mathrm{wt} \%$ ).

\section{The toughening mechanism}

The percolation by hydrogen bonds seems to be a good compromise between stiffness and toughness conferred to the composites. If the interactions between nanofibers are too strong, they are not able to slide past each other during plastic deformation, resulting in brittle materials even though they show high modulus. Bacterial cellulose is secreted extracellularly by Acetobacter species, forming a networked structure of nanofibers of pure cellulose that are straight, continuous, and dimensionally uniform. These nanofibers of animal origin are already in the form of individualized nanofibers, although they are connected along the length as the bacteria spin nanofibers continually even during cell division. As the stress-strain curves of Fig. 3 show, bacterial cellulose-based nanocomposites have a Young's modulus of up to $28 \mathrm{GPa}$ and strength exceeding $400 \mathrm{MPa}$, but the strain at fracture is small compared to composites based on cellulose nanofibers [13]. The loose and hairy nature of nanofibers allow them to be drawn and straightened during deformation, and by sliding past one another due to moderately strong hydrogen bonds, they absorb significant amount of energy before failure. Bacterial cellulose nanofibers on the other hand are already straight and continuously connected lengthwise, offering few options for a toughening mechanism.

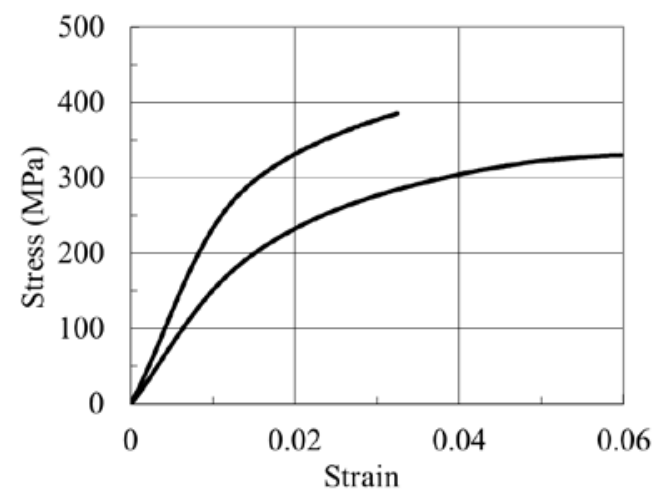

Fig. 3. Typical flexural stress-strain curves of bacterial cellulose/phenolic resin (a) vs. cellulose nanofiber/phenolic resin nanocomposites (b) (resin content of both materials: 2.7wt\%).

As much as cellulose nanofibers deliver tougher composites relative to bacterial cellulose or cellulose microfiber counterparts, the strain at fracture is not significant in absolute values. Alkali treatment, among other chemical treatments, is one of the usual ways to improve fiber-matrix adhesion in natural fiber-based composites. However, another interesting application was reported by Goda et al. [14], who made composites with ramie fibers treated with alkali solution. They found that a significant increase in toughness with no decrease in strength. The explanation for this phenomenon 
takes into account the structure of the cell wall of natural fibers, but the question that remained is if the same effect would come out of nanofibers, whose original cellular structure no longer exist. The cellulose nanofibers were treated with a $5 \mathrm{wt} \%$ and a strong $20 \mathrm{wt} \% \mathrm{NaOH}$ aqueous solutions [15]. After composition with phenolic resin, the same increase in toughness could be observed for the composites made with nanofibers treated with the strong alkali solution, as depicted in Fig. 4. The possible explanation at the nanoscale level was that cellulose nanofibers contract longitudinally [16] due to an entropic elastic mechanism at the amorphous portions [17], and extend when under load, however nothing could be inferred about the changes in the interactions between the nanofibers. The only sure conclusion was that only a strong alkali treatment of fibers can increase the toughness of composites.

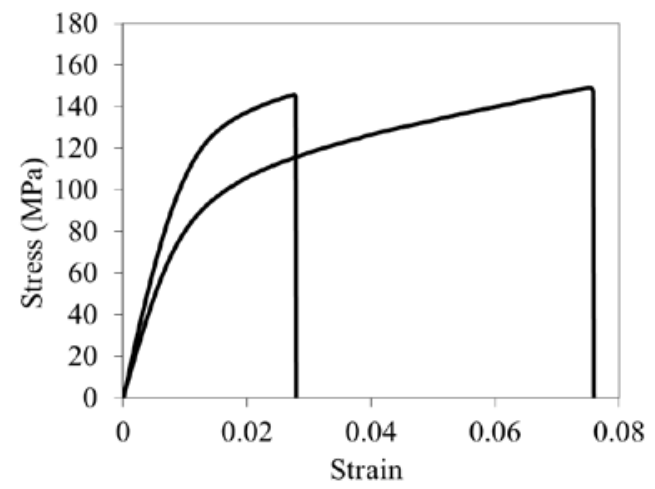

Fig. 4. Typical tensile stress-strain curves of untreated cellulose nanofiber/phenolic resin nanocomposite (a) (resin content: $13.6 \mathrm{wt} \%$ ) vs. alkali treated cellulose nanofiber/phenolic resin nanocomposite (b) (resin content: 18.5wt\%).

\section{The challenges to produce cellulose nano- composites}

All of the mentioned nanocomposites were carefully prepared so to preserve the percolated nanofiber structure, whether by evaporation of aqueous suspensions or by obtaining paper-like sheets of nanofibers for posterior resin impregnation. In this way the good dispersion of nanofibers inside the matrix phase was assured. The proper dispersion of nanofibers is one of the critical points to obtain reinforcements in cellulose nanocomposites as demonstrated by Takagi and Asano [18]. They prepared green composites by mixing cellulose nanofiber aqueous suspension and starch dispersed in water, by different agitation methods. One used a domestic blender for a short time while the other was by a low speed stirring for a long period of time. Both composites were obtained by film casting and tensile tests revealed that slow stirring delivered composites with higher modulus, strength, and densities than mixing by a blender. The observation of the nanostructure showed that slow stirring results in homogeneously dispersed nanofibers as well. Processing condition plays an important role in nanofiber dispersion even when carried out in water medium. Even more difficult is to achieve dispersion of hydrophilic cellulose nanofibers in hydrophobic polymer melts, and compounding processes of cellulose nanocomposites have seen very little success so far.

Another unresolved issue concerning cellulose nanofibers is the excessively high cost of the nanofibers themselves. The extraction process is still time and energy intensive, with low production yields. Another limitation is the necessity of expensive and specialized devices to accomplish fibrillation, limiting the access to the nanofibers and therefore confining the research and development of new materials to few research groups in the world. If a lower cost and simpler process of nanofiber extraction were developed, the research for new applications of cellulose nanofibers would accelerate and eventually commodity products would become a reality. Uses in transportation like automobiles, airplanes and boats would be very much benefitted by savings due to efficiencies in fuel consumption, through the use of lighter but strong composites based on sustainable resources. In the past, wood served well in many purposes as a material, and these new cellulose nanocomposites would be a plant derived alternative with tailored properties for each application.

\section{References}

[1] T. Nishino, K. Takano and K. Nakamae, "Elastic Modulus of the Crystalline Regions of Cellulose Polymorphs," Journal of Polymer Science Part B - Polymer Physics 33, (1995) pp1647-1651. 
[2] W. Helbert, J. Y. Cavaille and A. Dufresne, "Thermoplastic Nanocomposites Filled with Wheat Straw Cellulose Whiskers," Part I: Processing and Mechanical Behavior, Polymer Composites, 17, (1996) pp604-611.

[3] A. Boldizar, C. Klason, J. Kubat, P. Naslund and P. Saha, "Prehydrolyzed Cellulose as Reinforcing Filler for Thermoplastics,” International Journal of Polymeric Materials 11 (1987) pp229-262.

[4] L. A. Berglund (Ed.: M. A. D. Mohanty, M. Misra and L. T. Druzal), "Natural Fibers, Biopolymers, and their Biocomposites,” (2005) CRC Press LLC.

[5] V. Favier, H. Chanzy and J. Y. Cavaille, "Polymer Nanocomposites Reinforced by Cellulose Whiskers," Macromolecules 28, (1995) pp6365-6367.

[6] V. Favier, G. R. Canova, J. Y. Cavaille, H. Chanzy, A. Dufresne and C. Gauthier, "Nanocomposite Materials from Latex and Cellulose Whiskers," Polymers for Advanced Technologies 6, (1995) pp351-355.

[7] A. Dufresne and M. R. Vignon, "Improvement of Starch Film Performances Using Cellulose Microfibrils,” Macromolecules 31, (1998) pp2693-2696.

[8] M. A. S. A. Samir, F. Alloin, M. Paillet and A. Dufresne, "Tangling Effect in Fibrillated Cellulose Reinforced Nanocomposites,” Macromolecules 37, (2004) 4313-4316.

[9] H. Yano and S. Nakahara, "Bio-composites Produced from Plant Microfiber Bundles with a Nanometer Unit Web-like Network,” Journal of Materials Science 39, (2004), pp1635-1638.

[10]A. N. Nakagaito and H. Yano, "Novel Highstrength Biocomposites Based on Microfibrillated Cellulose Having Nano-order-unit Weblike Network Structure,” Applied Physics A Materials Science and Processing 80, (2005) pp155-159.

[11]A. N. Nakagaito an H. Yano, "The Effect of Morphological Changes from Pulp Fiber To- wards Nano-scale Fibrillated Cellulose on the Mechanical Properties of High-strength Plant Fiber Based Composites,” Applied Physics A Materials Science and Processing 78, (2004) pp547-552.

[12]H. Takagi, "Strength Properties of Cellulose Nanofiber Green Composites,” Key Engineering Materials 462-463, (2011) pp576-581.

[13]A. N. Nakagaito, S. Iwamoto, H. Yano, "Bacterial Cellulose: the Ultimate Nano-scalar Cellulose Morphology for the Production of Highstrength Composites," Applied Physics A - Materials Science and Processing 80, (2005) pp93-97.

[14]K. Goda, M. S. Sreekala, A. Gomes, T. Kaji and J. Ohgi, "Improvement of Plant Based Natural Fibers for Toughening Green Composites - Effect of Load Application During Mercerization of Ramie Fibers," Composites Part A: Applied Science and Manufacturing 37, (2006) pp2213-2220.

[15]A. N. Nakagaito and H. Yano, "Toughness Enhancement of Cellulose Nanocomposites by Alkali Treatment of the Reinforcing Cellulose Nanofibers,” Cellulose 15, (2008) pp323-331.

[16]Y. Ishikura and T. Nakano, "Contraction of the Microfibrils of Wood Treated with Aqueous $\mathrm{NaOH}$ : Evidence from Changes in the Anisotropy of the Longitudinal and Transverse Swelling Rates of Wood,” Journal of Wood Science, 53, (2007) pp175-177.

[17]T. Nakano, J. Sugiyama and M. Norimoto, "Contractive Force and Transformation of Microfibril with Aqueous Sodium Hydroxide Solution for Wood,” Holzforschung 54, (2000) pp315-320.

[18]H. Takagi and A. Asano, "Effects of Processing Conditions on Flexural Properties of Cellulose Nanofiber Reinforced "Green” Composites", Composites Part A: Applied Science and Manufacturing 39, (2008) pp685-689. 